\title{
ACRODAT $^{\oplus}$ and AcroVoice: an insight into a holistic approach to the management of acromegaly
}

\author{
Mark Lundie $^{1} \cdot$ Jill Sisco $^{2} \cdot$ Aart J. van der Lely $^{3}$
}

Published online: 9 October 2019

(c) The Author(s) 2019

To the Editor,

We read the Letter to the Editor by Wang and Xing, 2019, entitled "AcroVoice: the controversial values in reflecting acromegaly disease activity" with some concern [1]. The authors misinterpreted the ACRODAT ${ }^{\circledR}$ study, and there is a need to correct this. Wang and Xing, 2019 presented a critique of the ACRODAT ${ }^{\circledR}$ study, not AcroVoice, and did not even reference the ACRODAT ${ }^{\circledR}$ paper [2] in their Letter to the Editor [1]. Moreover, Wang and Xing expressed concerns regarding the "rationality of the five parameters of ACRODAT®" [1].

As has been published, ACRODAT ${ }^{\circledR}$ was developed with expert opinion and validated in a rigorous study [2]. The resulting five dimensions (tumor status, insulin-like growth factor I [IGF-I] levels, comorbid conditions [diabetes, sleep apnea, and cardiac disease], symptoms, and quality of life [QoL]) offer a fast, simple tool to obtain an overview of patients' disease and, when needed, help make informed treatment decisions [2]. The dimensions of ACRODAT ${ }^{\circledR}$ were carefully chosen to produce a balance between being practical to use and inclusive with respect to its power to reflect all of the important information on disease activity in acromegaly. Moreover, the dimensions of ACRODAT ${ }^{\circledR}$ are used by patients to gauge the severity of their disease, highlighting the importance of all components of acromegaly in the assessment of their condition. The inclusion of additional data or dimensions was evaluated by the expert panel; it was believed this did not add to the tool, and additionally, may confound potential issues in disease management.

Mark Lundie

mark.lundie@pfizer.com

Pfizer Canada, Kirkland, QC, Canada

2 Acromegaly Community, Grove, OK, USA

3 Erasmus University Medical Center, Rotterdam, The Netherlands
With regard to the AcroVoice study, Wang and Xing raised concerns about the variability in patients' responses to the discrete choice experiment (DCE) questions [1]. However, AcroVoice also employed a rigorous study design and the responses from the sample size of 100 patients exhibited a normal distribution, as would be expected from a large cohort, for which a pre-determined statistical analysis was employed to ascertain the robustness of the responses [3]. Moreover, Wang and Xing raised concerns that the patients' responses or selections were too subjective since no specific values were used to define "slight" or "significant" abnormalities [1]. Again, the assumption is not accurate, and in fact, specific values were ascribed to slight or significant abnormalities for each parameter.

ACRODAT ${ }^{\circledR}$ and AcroVoice offer insights into a holistic approach to the management of acromegaly [2, 3]. Importantly, the AcroVoice study considered the patients' perspectives surrounding their care and highlighted the strong desire of patients to have an active role in their disease management [3]. The AcroVoice study showed that patients with acromegaly valued both clinical factors and patient-centered factors. The study emphasized the importance of personalized care [3]. The AcroVoice study also showed that shared decision-making between patients and their doctors is important in making treatment decisions [3]. To support this conversation between patients and their healthcare providers, a Plain Language Summary (https://www.acromegalywest. com/blog) was developed.

Acknowledgements Medical writing support was provided by Neel Misra MSc, CMPP, of Engage Scientific Solutions and was funded by Pfizer.

\section{Compliance with ethical standards}

Conflict of interest ML is a full-time employee of and declares stock holdings or stock options from Pfizer. JS has received speaker fees from Pfizer and presented content from the AcroVoice research project 
at the Pituitary Society meeting. AJvdL has received consultancy and speaker fees from Pfizer.

Ethical approval This article does not contain any studies with human participants performed by the author.

Open Access This article is distributed under the terms of the Creative Commons Attribution 4.0 International License (http://creativeco mmons.org/licenses/by/4.0/), which permits unrestricted use, distribution, and reproduction in any medium, provided you give appropriate credit to the original author(s) and the source, provide a link to the Creative Commons license, and indicate if changes were made.

\section{References}

1. Wang Z, Xing B (2019) AcroVoice: the controversial values in reflecting acromegaly disease activity. Pituitary 22:443-444
2. van der Lely AJ, Gomez R, Pleil A, Badia X, Brue T, Buchfelder M, Burman P, Clemmons D, Ghigo E, Jørgensen JOL, Luger A, van der Lans-Bussemaker J, Webb SM, Strasburger CJ (2017) Development of ACRODAT ${ }^{\circledR}$, a new software medical device to assess disease activity in patients with acromegaly. Pituitary 20:692-701

3. Jackson Y, Flood E, Rhoten S, Janssen EM, Lundie M (2019) AcroVoice: eliciting the patients' perspective on acromegaly disease activity. Pituitary 22:62-69

Publisher's Note Springer Nature remains neutral with regard to jurisdictional claims in published maps and institutional affiliations. 